\title{
El debate sobre la digitalización y la robotización del trabajo (humano) del futuro: automatización de sustitución, pragmatismo tecnológico, automatización de integración y heteromatización Debates on digitization and robotization of (human) work of the future: replacing automation, technological pragmatism, augmentation and heteromation
}

\author{
Arturo Lahera Sánchez \\ Universidad Complutense de Madrid, España \\ alaheras@ucm.es
}

Received / Recibido: 20/10/2020

Accepted / Aceptado: 01/12/2020

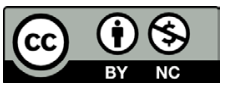

\section{RESUMEN}

Se realiza una revisión al 'estado del arte' de los debates sobre un supuesto desempleo tecnológico masivo en el futuro como consecuencia de la difusión de procesos de digitalización y robotización con las nuevas tecnologías de la Industria 4.0. Se presentan y discuten cuatro enfoques alternativos, pero complementarios, sobre la nueva ola de automatización: automatización de sustitución, pragmatismo tecnológico, automatización de integración y heteromatización. Desde una perspectiva constructivista sobre la tecnología, se cuestionan críticamente algunos argumentos dominantes sobre la desaparición masiva del empleo del futuro, apostando por señalar su improbabilidad, mostrando la estratificación de g rupos perdedores y ganadores de la digitalización y la robotización, junto a las dificultades para formar a los primeros para su inclusión digital. Por último, se defiende la importancia de hacer visible el oculto trabajo humano que sustenta los procesos automatizados de la producción digitalizada y la posibilidad de reforzar las cualificaciones y competencias del factor humano, su recualificación a través de una automatización ergonómica.

Palabras clave: digitalización, robotización, industria 4.0, heteromatización, desempleo tecnológico.

\begin{abstract}
A review is made about the 'state of the art' of the debates on an alleged massive technological unemployment in the future as a consequence of the diffusion of digitization and robotization processes with the new technologies of the Industry 4.0. Four alternative but complementary approaches about the present new wave of automation are presented
\end{abstract}

*Autor para correspondencia / Corresponding author: Arturo Lahera Sánchez, alaheras@ucm.es

Suggested citation / Sugerencia de cita: Lahera Sánchez, A. (2021). El debate sobre la digitalización y la robotización del trabajo (humano) del futuro: automatización de sustitución, pragmatismo tecnológico, automatización de integración y heteromatización. Revista Española de Sociología, 30 (3), a66 https://doi.org/10.22325/fes/res.2021.66 
El debate sobre la digitalización y la robotización del trabajo (humano) del futuro: automatización de sustitución, pragmatismo tecnológico, automatización de integración y heteromatización

and discussed: replacing automation, technological pragmatism, augmentation, and heteromation. From a constructivist perspective on technology, some dominant arguments about the massive disappearance of future employment are critically questioned, pointing out its improbability, showing the stratification of losers and winners from digitization and robotization, highlighting the difficulties to train those first ones for their digital inclusion. Finally, it is defended the importance of making visible the hidden human work supporting automated processes of digitized production and also the possibility of reinforcing the qualifications and competencies of the human factor, its reskilling, through an ergonomic automation.

Keywords: digitization, robotization, industry 4.0, heteromation, technological unemployment.

En el último lustro se ha difundido mediáticamente una nueva ola de 'ansiedad por la automatización': el miedo a un inevitable y mayoritario desempleo tecnológico futuro, en el que la difusión de la inteligencia artificial (IA), la digitalización de los procesos laborales de fabricación de bienes o de prestación de servicios, así como su robotización, irán continuamente haciendo desaparecer el empleo humano. Discurso 'tecno-pesimista' o 'tecno-fatalista' basado en una continua presentación de nuevas tecnologías digitales o robóticas que realizan tareas sin supervisión, que hasta ahora solo eran realizadas por el trabajo humano (automóviles que 'conducen' autónomamente', robots antropomórficos que atienden restaurantes o cocinan, ordenadores que realizan todo tipo de tareas autónomamente o diagnostican enfermedades...). Este fatalismo se refuerza además con la 'socialización' de estudios e investigaciones (econométricas) que anticipan la desaparición de casi la mitad de los empleos en las próximas décadas, porque sus tareas son fácilmente automatizables: investigaciones que, a pesar de que sus resultados son refutados y contraargumentados, siguen siendo el referente mediático hegemónico, mientras que los últimos apenas aparecen referenciados.

Además, este discurso del 'fin del trabajo humano' se intensifica al no haber superado todavía, especialmente en el mercado de trabajo español, los efectos de destrucción de empleo por lo que es una 'onda larga' de (aún más) vulnerabilidad laboral desde el inicio de la Gran Recesión en 2008 , con la profundización de las inercias de precarización, de degradación de las condiciones de empleo, de devaluación salarial e incremento de la desafiliación de la ciudadanía, con lo que el discurso de un cercano ‘apocalipsis robótico' tiene más facilidad para aceptarse acríticamente ${ }^{1}$.

Por ejemplo, en la Encuesta sobre Percepción Social de la Ciencia y la Tecnología de 2018, realizada por la Fundación Española para la Ciencia y la Tecnología (FECYT), recoge que alrededor del $46 \%$ de las personas entrevistadas sitúan la robotización como una aplicación tecnológica con muchos o bastantes riesgos (frente a un $40 \%$ que la consideran beneficiosa); mientras que más del $38 \%$ consideran también que la inteligencia artificial tiene riesgos, frente al $43 \%$ que consideran que tiene más beneficios (Lobera y TorresAlbero, 2019). También la III Encuesta de Percepción Social de la Innovación en España, realizada por la Fundación Cotec en 2020, mostraba que alrededor de dos tercios de

\footnotetext{
1 Algún ejemplo: en 2015, lo que iba a ser la cadena de restaurantes automatizados y sin empleados de sala EATSA, en el área de San Francisco, (California) protagonizó decenas de noticias y reportajes sobre cómo iba a revolucionar el trabajo de restauración mediante un proceso robotizado (robo-restaurant), en el que los clientes realizaban sus pedidos a través de terminales digitales que, tras ser procesados (y preparados, eso sí, en cocinas ocultas... con 'humanos'), eran recogidos directamente por esos clientes tras abrir las puertas de unos compartimentos en los muros del restaurante, sin ningún tipo de interacción humana. Los restaurantes EATSA aparecían como un modelo que se iría difundiendo y sustituyendo el trabajo de camareras y camareros a partir de ese momento. Si la puesta en marcha de estos 'restaurantes automáticos' copó noticias en medios de todo tipo (prensa económica, revistas tecnológicas, blogs tecnológicos, enlaces en youtube...), su cierre definitivo en el verano de 2019 (meses antes de la aparición de la pandemia de covid-19), con suspensión de pagos, no tuvo apenas repercusión. EATSA ha cambiado de nombre a Brightloom, como empresa de software y tecnología de apoyo a la industria de restaurantes. Algo similar en este sector ha ocurrido con las cadenas Creator (un robot enorme fabricaba hamburguesas), CafeX (un robot preparaba cafés a sus clientes), Blendid (batidos preparados por robots), Zume (pizzas 'robóticas'), habiendo cerrado definitivamente (mayoritariamente) o dependen cada vez más de 'trabajadores humanos' para poder gestionar eficazmente el proceso (Canales, 2020).
} 
una muestra representativa de la población española, considera que la automatización sustituirá 'muchos' puestos de trabajo actuales por ordenadores o robots, mientras un $43 \%$ cree que estos podrán hacer tareas rutinarias, pero también creativas y un $47 \%$ afirma que los empleos perdidos por la automatización no se compensarán con la creación de nuevos empleos; al tiempo que alrededor de un tercio no se considera capacitado para competir en un mercado de trabajo digitalizado (Fundación COTEC, 2020).

Sin embargo, el debate investigador y académico plantea una mayor complejidad sobre los efectos sociales y laborales de las nuevas tecnologías digitales y robóticas de la actual Cuarta Revolución Industrial, con enfoques contrapuestos sobre las características de esta nueva fase de automatización, sobre la forma de 'enfrentarse' a ella, para intentar 'domesticarla' o simplemente para 'proteger' a aquellos grupos sociales (¿la mayoría?) que se pudieran enfrentar al desempleo tecnológico futuro.

Complejidad que puede ser presentada en cuatro enfoques alternativos de los que, como es habitual en la reflexión social y sociológica, se pueden obtener herramientas de interpretación que permiten defender una perspectiva 'pragmática' para explicar y comprender experiencias 'reales' de automatización digital o robótica en empresas y organizaciones que ‘sufren' la transformación digital de esta nueva etapa de implantación de dispositivos tecnológicos... o que se verán afectadas en el futuro (mejor cercano, si se pretende pronosticar o hacer prognosis con ciertas garantías de certeza).

\section{(A) AUTOMATIZACIÓN DE SUSTITUCIÓN: EL ENFOQUE DOMINANTE EN LOS DISCURSOS MEDIÁTICOS SOBRE EL FIN DEL TRABAJO (HUMANO) Y DEL EMPLEO:}

El primer enfoque de este debate es aquel que está siendo más exitoso mediáticamente y más difundido en los imaginarios sociales del último lustro (hasta la irrupción, verdaderamente 'disruptiva' de la pandemia de covid-19): el desempleo tecnológico masivo por la imparable aplicación de una (entendida como digitalización y robotización del trabajo y de los procesos productivos) automatización de sustitución del trabajo humano.

Esta automatización supone que la inteligencia artificial y la robótica avanzada de esta última Cuarta Revolución Industrial está permitiendo que muchas ocupaciones o puestos de trabajo puedan dejar de realizarse por el trabajo humano y pasen a ser realizados (¿de manera completa?) por esas nuevas tecnologías digitales, que, aunque requieran también la emergencia de nuevas ocupaciones para su programación o mantenimiento, no permitirán compensar la pérdida de empleos de baja o media cualificación, que son mayoritarios en los mercados de trabajo occidentales (Acemoglu y Restrepo, 2017; Brynjolfsson y McAfee, 2011; Doménech, García, Montáñez y Neut, 2018). De esta forma, esta automatización de sustitución está reemplazando al ser humano (replacing automation), especialmente a quienes desempeñan tareas rutinarias, repetitivas, de baja complejidad o simples (en movimientos y/o cálculos), estandarizadas o fácilmente estandarizables (que pueden ser traducidas a algoritmos informáticos programados en aplicaciones de software y en acciones de robots con sus 'actuadores'): mediante mecanismos que transfieren las tareas realizadas por el ser humano a los nuevos dispositivos tecnológicos (Acemoglu y Restrepo, 2017; Brynjolfsson y McAfee, 2011; Doménech et al., 2018).

La justificación de este enfoque de automatización de sustitución se basa continuamente en la predicción de 2013 (en el momento álgido de la crisis financiera internacional y con un intenso 'impacto' académico, sí, pero también mediático) de Frey y Osborne (2013), en la que aseguraban que hasta el $47 \%$ de los empleos de los Estados Unidos estaban en riesgo de desaparecer en alrededor de un par de décadas: de forma que, la introducción y difusión de la inteligencia artificial y de adquisición de robots implicaba directamente la desaparición de varios empleos por la instalación de una nueva unidad de estas tecnologías. Es cierto que si bien esa predicción ha sido criticada y refutada, 
El debate sobre la digitalización y la robotización del trabajo (humano) del futuro: automatización de sustitución, pragmatismo tecnológico, automatización de integración y heteromatización

como se señalará en breve, por otras investigaciones (Mishel y Bivens, 2017), este enfoque de sustitución tecnológica del trabajo humano en el futuro es apoyada en diversas obras (Ford, 2015; Ortega, 2016; Susskind, 2020), que siguen enfatizando la desaparición de esas ocupaciones rutinarias y de media o baja cualificación, enfatizando la potencialidad de las nuevas tecnologías digitales, el incremento continuo de su inteligencia artificial, para ir 'copando' cada vez nuevas tareas humanas, de manera más eficaz y más eficiente, más allá de la capacidad humana (World Economic Forum, 2016): “Las máquinas igualan o mejoran el rendimiento humano en múltiples actividades, incluso las que requieren capacidades cognitivas" (McKinsey Global Institute, 2017, p. 3).

La única opción es un pesimismo o fatalismo tecnológico por la ineluctable desaparición de millones de empleos. Aunque históricamente, a pesar de los temores del movimiento 'ludita' en la primera Revolución Industrial², toda nueva ola de innovación tecnológica en el desarrollo de la economía de mercado capitalista no ha ido acompañada real o empíricamente de la desaparición del empleo, sino de la creación de múltiples sectores productivos y de servicios que reemplazaron abundantemente las ocupaciones destruidas por la propia tecnología (la clásica destrucción creativa), en la Cuarta Revolución Industrial ya no ocurrirá así, puesto que los empleos descualificados basados en tareas simples y rutinarias serán realizados por la inteligencia artificial digital y robotizada y sólo se crearán, en mucha menor medida, empleos de alta cualificación, basados en la adquisición educativa de competencia y conocimientos matemáticos e informáticos, que no estarán al alcance de la mayoría de la ciudadanía (competencias STEM: credenciales educativas en ciencia, tecnología, ingeniería, matemáticas...).

Desde este enfoque se justifican políticas públicas tanto conservadoras (centradas en impedir las limitaciones 'estatales' de protección social que desincentiven la innovación tecnológica y la implantación generalizada de la Al y la robótica avanzada, socavando las ganancias de productividad capturadas por las empresas y sus accionistas, situando la responsabilidad en la actividad 'proactiva' de los posibles afectados en su voluntad de formación y emprendimiento), como socialdemócratas (que apuestan por invertir colectivamente en la recualificación del trabajo humano que pueda ser 'desplazado' por la digitalización, planteando nuevas figuras impositivas sobre las unidades de robot, una 'robotasa'...), hasta propuestas desde diversos grados de socialismo, desde la defensa de una 'renta básica universal' incondicional para evitar los efectos destructores de la sociabilidad colectiva de ese inevitable desempleo tecnológico, hasta la posibilidad de una sociedad socialista definitiva (Benanav, 2019). El 'apocalipsis robótico' es inevitable en muy pocos años o décadas (más bien a través de todo tipo de dispositivos digitales de inteligencia artificial, no necesariamente antropomórficos...) y solo queda (en un nuevo determinismo tecnológico que parecía haber sido superado por la difusión de los 'estudios sociales de la tecnología' y de los enfoques de la 'construcción social de la tecnología'...) que nuestras sociedades se adapten a las características sustitutivas del trabajo humano de esta Cuarta Revolución Industrial. No habrá trabajo suficiente para toda la población, punto: “¿Habrá suficientes empleos para todo el mundo en el siglo veintiuno? No" (Susskind, 2020, p. 5).

\section{(B) UN ENFOQUE PRAGMÁTICOSOBRE LA DIGITALIZACIÓN Y LAROBOTIZACIÓN: PERDEDORES Y GANADORES DE LA AUTOMATIZACIÓN EN UN FUTURO CON TRABAJO HUMANO (AUNQUE DIFERENTE):}

A partir de este enfoque, y como crítica a sus asunciones, se ha articulado una interpretación alternativa: un enfoque pragmático sobre los nuevos procesos de automatización. Su punto de partida, también con base en investigaciones econométricas

\footnotetext{
2 Temores que fueron reales durante un buen número de décadas, con un intenso desempleo tecnológico entre 1770 y 1840 , con el deterioro salarial, de las condiciones de vida de la mano de obra británica, junto con la 'apropiación' (casi) completa de las ganancias de productividad por los industriales (el 'capital') gracias a las nuevas máquinas textiles que sustituían el trabajo humano: siguiendo la discutida 'pausa de Engels' difundida por el historiador económico Robert Allen (2007).
} 
(por tanto, de nuevo alejadas de un conocimiento de terreno sobre cómo se implantan organizativa y realmente las tecnologías digitales de la Industria 4.0), es cuestionar el inevitable desempleo tecnológico de las predicciones 'apocalípticas' del enfoque de la automatización de sustitución. Frente a estas, el pragmatismo señala que la difusión de la digitalización, la inteligencia artificial y la robótica avanzada no está provocando desempleo generalizado (Arntz, Gregory y Zierhan, 2016), sino incluso un incremento del empleo total mundial (previamente a la pandemia del coronavirus sars-cov-2). De hecho, hay que enfatizar que las economías con una mayor penetración e instalación de estas tecnologías (por ejemplo, especialmente robots) han logrado pleno empleo: destacando Alemania y Corea del Sur, por ejemplo (International Federation of Robotics, 2018).

Por tanto, el pragmatismo aboga, en relación con las causas de un actual y futuro desempleo, por complementar la variable de la tecnología de automatización y su posible destrucción de ocupaciones (más bien, de parte de sus tareas: no de ocupaciones completas), con variables como la exposición a la competencia globalizada (procesos de deslocalización productiva, 'guerras comerciales', emergencia de nuevos mercados de bienes y servicios, nuevas demandas de consumo...), llegando a la conclusión (y prognosis) que el efecto combinado de todas ellas tienen más importancia explicativa que la digitalización y la robotización: "La literatura revisada no es concluyente sobre si los robots incrementan o reducen el empleo ${ }^{3 "}$ (Klenert, Fernández-Macías y Antón, 2020, p. 11).

De esta forma, el enfoque pragmático duda, frente al alarmismo del enfoque de sustitución (Van Barneveld y Janson, 2017), del desempleo tecnológico digital y de la inteligencia artificial masivos y generalizados: ni ansiedad por la automatización, ni por la inevitable singularidad tecnológica de la ciencia-ficción ${ }^{4}$, ni por aquel apocalipsis humano provocado por los robots.

Sin embargo, ¿en qué basa este enfoque su 'pragmatismo'? Por un lado, en compartir y reconocer que sí, que la Cuarta Revolución Industrial también se basa en procesos de automatización de sustitución, pero que, sin embargo, no se generalizarán a centenares de ocupaciones (sino más bien a parte de sus tareas; Lahera Sánchez, 2019), sino a algunas de ellas, de forma que, efectivamente, se perderán empleos definitivamente, pero que serán reemplazados por otros nuevos empleos, en nuevos sectores, en nuevas tareas (programación, mantenimiento, gestión de procesos digitales...) y nuevos productos y servicios (como ha ejemplificado el sector del consumo de ocio, de comunicación personal y telecomunicaciones, de actividades de cuidados...). La clave pragmática es identificar qué ocupaciones tienen más probabilidad de ser realmente 'sustituidas' o 'reemplazadas' digitalmente (más bien cuáles o qué proporción de sus tareas pueden ser automatizadas) para intervenir en la reducción de los efectos negativos de ese desempleo tecnológico 'sectorial', a través de un (¿mítico y utópico?) reciclaje profesional, formación continua y protección social a los desplazados por la Industria 4.0 y la inteligencia artificial (World Economic Forum, 2018).

Por otro lado, este pragmatismo se basa en apostar metodológicamente no por generalizar las posibles, 'increíbles' y supuestas capacidades productivas de las nuevas tecnologías digitales (desde coches y camiones autónomos que inundarán ¿en breve? nuestras carreteras; hyperloops que recorrerán centenares de kilómetros en unos

3 Estos autores señalan, analizando la situación actual en Europa, que la continua instalación de robots en las dos décadas entre 1995 y 2015 ha implicado un mantenimiento o ligero incremento del empleo: llegando en algunos sectores a implicar que por cada robot instalado se crean hasta 5 empleos en la economía general o, lo que es lo mismo, la incorporación adicional de un robot por cada 1000 trabajadores correlaciona con un incremento de un 1'31\% del empleo total (página 31).

4 La singularidad tecnológica es una predicción (¿deseo?) de la ciencia-ficción en que la acumulación de dispositivos de inteligencia artificial acabará creando, por su conexión, una inteligencia artificial general capaz de autorreplicarse y mejorarse recurrentemente, fabricando robots y algoritmos que irán conquistando cada vez más tareas y procesos productivos, que escaparán al control humano... que acabaría sojuzgado por esa inteligencia artificial fuerte. Mito cultural que está en la base de múltiples obras narrativas muy exitosas socialmente: entre otras, HAL 9000, el gólem, los replicantes en Blade Runner (y la novela original de Philip K. Dick), los robots 'independizados' y conflictivos en Westworld (tanto en su primera versión literaria y cinematográfica de Michael Crichton en 1973, con el inicio del incumplido miedo a la automatización desde finales de la década de 1960, como en la serie de televisión desde 2016), o la inteligencia 'antihumana' de Skynet en la saga Terminator... 
El debate sobre la digitalización y la robotización del trabajo (humano) del futuro: automatización de sustitución, pragmatismo tecnológico, automatización de integración y heteromatización

pocos minutos; impresoras 3D que fabricarán piezas metálicas, y no solo de plástico, sin necesidad de largos procesos de mecanizado; internet industrial de las cosas, cuyos sensores permitirán conectar máquinas, computadoras y herramientas en una, ¿otra vez anhelada?, fábrica automática sin trabajo humano $\left.0^{5} ..\right)$, sino en enfatizar y recordar que muchas de estas innovaciones están todavía en un nivel de desarrollo que las acercan más a ser prototipos que a artefactos tecnológicos directamente aplicables y que puedan ser ya (o a corto plazo) rentables: "Los robots no son ni tan rápidos ni tan fluidos como un trabajador humano bien formado a toda capacidad" (Brynjolfsson y McAfee, 2011, p. 58). Todo lo cual implica que su difusión vaya a ser, con más probabilidad, más lenta de lo 'vaticinado' (décadas más que años: es más una subida de la marea, que un tsunami; Dellot, 2018) y, por tanto, que su efecto global sobre la sustitución y destrucción de empleo pueda ser mucho menor que el anticipado por el enfoque de la automatización de sustitución: "Solo porque algo pueda ser automatizado teóricamente no significa que vaya a ser económicamente o políticamente viable en la práctica" (PriceWaterhouseCoopers, 2018 , p. 1). Por ejemplo, las tecnologías más 'famosas' de la industria 4.0, como la robótica avanzada, la fabricación aditiva o impresión 3D, la internet industrial de las cosas, o la realidad virtual/ampliada están todavía en su infancia, afrontando también barreras técnicas (de integración con los 'tradicionales' sistemas informáticos de las empresas, costes de instalación, operación y mantenimiento), financieras (requieren fuertes inversiones), que ralentizan su implantación (Stewart, Deshpande, Hoorens y Gunasekara, 2019): “No hay duda de que los robots del futuro serán capaces de ejecutar tareas extraordinarias, pero actualmente, sus aplicaciones comerciales han sido limitadas. El futuro está todavía probablemente algo lejos en el tiempo y llegará gradualmente" (Eurofound, 2019, p. 42). Por ejemplo, es importante saber que el coste de instalación, formación y mantenimiento de una nueva tecnología o dispositivo puede suponer de tres a cinco veces el coste del propio dispositivo (Brynjolfsson y McAfee, 2015). Con lo que la decisión organizativa o empresarial suele ser más cautelosa de lo que los discursos mediáticos suelen difundir (¿exageración tecnológica?), lo que está implicando en la práctica real de las empresas una difusión escasamente acelerada: "Si todos los puestos de trabajo susceptibles de ser automatizados lo fueran efectivamente, la inversión requerida sería de una proporción tan grande del PIB que es simplemente irreal desde una perspectiva macroeconómica" (Eurofound, 2019, p. 38).

La clave estaría, en términos de políticas públicas y de intervención social, en cartografiar qué empleos, ocupaciones, tareas, sectores, perfiles profesionales y personas se están convirtiendo ya y a corto plazo en perdedores o perdedoras de las aplicaciones ya (más o menos) maduras de la digitalización, la robotización y la inteligencia artificial, para reducir los efectos de su desempleo tecnológico sectorial y posible desafiliación social. Cada vez es más claro que esos perfiles perdedores son fundamentalmente los basados en tareas semi y descualificadas, simples, estandarizadas, rutinarias, con alta regularidad en contextos controlados $\mathrm{y}$, por ello, fácilmente automatizables para su desempeño digital (Nedelkoska y Quintini, 2018, pp. 6-7): que han sido y son 'usurpados' por estas nuevas tecnologías (task encroachment; Susskind, 2020). Precisamente las que han sustentado los puestos de trabajo de las clases medias, de cuellos azules y blancos, durante la onda tecnológica de la segunda posguerra mundial, amenazadas por la deslocalización globalizada, por su industrialización y por la propia digitalización (conectándolas con la emergencia de movimientos populistas reaccionarios en las economías desarrolladas: "los perdedores de la tecnología están pidiendo cambios": Frey, 2019, p. 26).

Ahora bien, teniendo en cuenta este previsto mayor desempleo tecnológico en trabajadores y trabajadoras sin competencias digitales o con cualificaciones básicas, sería de esperar que manifestaran una mayor oposición hacia la inteligencia artificial

\footnotetext{
5 Por ejemplo, respecto a la internet industrial de las cosas, sigue habiendo dudas de que ya sea o vaya a ser una industria dominante, por los requisitos de alta inversión que requiere a largo plazo: “las declaraciones (...) se basan sólo en expectativas expresadas en la literatura o por expertos. Sólo unos pocos casos han demostrado sus efectos en el trabajo" (Scholten, 2017, p. 28). En relación con la fabricación aditiva o impresión $3 D$, sigue siendo mucho más costosa (por pieza) que cualquier otro método de mecanizado o conformación tradicionales (Van Barneveld y Janson, 2017).
} 
y sus aplicaciones, con las que están ya compitiendo en el mercado de trabajo para el mantenimiento de sus empleos y tareas. Sin embargo, algunos resultados empíricos no muestran ese mayor rechazo respecto al mantenido por otros perfiles sociales y laborales (Lobera, Fernández Rodríguez y Torres-Albero, 2020). Una de las hipótesis que podrían explicar este fenómeno estaría relacionada con que parece también conocido que la IA y los robots no pueden realizar eficaz y rentablemente (¿todavía?) puestos de trabajo de baja cualificación, pero basados en tareas y movimientos complejos, no estandarizables, irregulares, no rutinarios, que requieren conocimientos tácitos, especialmente en contextos de incertidumbre productiva con múltiples incidencias (construcción, servicios personales, servicios 'domésticos', trabajos de cuidado...), lo que puede facilitar una 'percepción' de cierta protección frente a las tecnologías digitales por parte de quienes desempeñan esos puestos difíciles de automatizar (Pfeiffer, 2018b; Lahera Sánchez, 2019, p. 265), a pesar de ser descualificados.

Frente a esos posibles grupos perdedores, se van estructurando (y estratificando socialmente) perfiles ganadores de la digitalización y robotización, aquellos con la formación y la experiencia para el diseño, la programación y el mantenimiento de los algoritmos (que no se olvide son artefactos que actúan gracias a su construcción social por diseñadores que les incorporan sus imaginarios ideológicos y sesgos): "La máquina será un demonio para unos, los sustituirá, mientras que, al contrario, será un ángel para otros, los complementará6" (Hidalgo, 2018, p. 14).

El enfoque pragmático, aunque también el enfoque de automatización de sustitución, defienden la prognosis de que los ganadores del proceso están siendo y serán aquellos perfiles con formación superior (de formación profesional o universitaria) en aquellas áreas STEM, con capacidad creativa para convertir e interpretar los datos (big data) capturados por la inteligencia artificial en procedimientos de mejora productiva (smart data: "pasar de un mar de datos a un mar de conocimientos", Ortega, 2016, p. 75), de programación de los algoritmos, razonamiento complejo, percepciốn y manipulación de procesos no estructurados difícilmente programables (Nedelkoska y Quintini, 2018): "conocimientos extensos de los procesos técnicos pero también capacidades de liderazgo y de resolución de problemas, así como atributos relacionados con el temperamento" (Eurofound, 2019, p. 48), para gestionar una tecnología bajo presión (pero ‘elegantemente': grace under pressure).

De hecho, la relación o el (des)equilibrio entre los perfiles profesionales y los puestos de trabajo perdedores y ganadores suponen la verdadera 'fractura tecnológica' a resolver. Tanto el enfoque de sustitución como el enfoque pragmático dan por hecho, o no problematizan, que la inversión formativa permitirá que quienes pierdan su empleo por la digitalización o por la incorporación de la robótica podrán lograr 'conectarse' con los perfiles ganadores gracias a la formación a lo largo de la vida laboral (como ‘obligación meritocrática', proactiva y emprendedora...; Serrano y Fernández Rodríguez, 2018), en una especie de 'reciclaje' competencial y credencial que evite su desafiliación definitiva. Axioma que es más que dudoso en el futuro si se plantea qué probabilidad real existe en que trabajadores y trabajadoras con bajos niveles de cualificación general, con escasas competencias digitales aprendidas, con conocimientos empíricos o experienciales en puestos de trabajo que han requerido limitadas cualificaciones efectivas, puedan adquirir, casi inmediatamente, las exigentes competencias digitales superiores que requieren los perfiles profesionales ganadores de la automatización 4.0. Más bien ya están siendo excluidos (operadores descualificados y/o veteranos; Degryse, 2016, p. 48): "Para estos trabajadores, la educación y la formación ofrecidas como soluciones en la narrativa dominante sobre los robots será inadecuada, al igual que fue inadecuada para ayudar a los trabajadores industriales manufactureros desplazados a lo largo de las últimas décadas" (Mishel y Bivens, 2017, p. 4).

\footnotetext{
6 Mientras sus propios conocimientos, competencias y habilidades digitales no sean también automatizadas, a medida que puedan ser estandarizadas, convertidas en rutinas y en nuevos algoritmos, en una carrera interminable entre el factor humano y la 'máquina' (Lahera Sánchez, 2006, pp. 282-305).
} 
El debate sobre la digitalización y la robotización del trabajo (humano) del futuro: automatización de sustitución, pragmatismo tecnológico, automatización de integración y heteromatización

La probabilidad de que los posibles perdedores acaben en un proceso de movilidad descendente en empleos y ocupaciones descualificadas del sector servicios, con una devaluación salarial y una degradación de sus condiciones de empleo es mucho mayor: como ha ocurrido, por cierto, en las últimas décadas con los también perdedores de la deslocalización y la desindustrialización de las economías desarrolladas por la globalización (frente a las clases medias ganadoras de esos procesos en las economías del subdesarrollo): "No es fácil para los camioneros reciclarse como programadores (...). Muchas personas no van a conseguir ciertas cualificaciones" (Susskind, 2020, p. 154). De la amplitud definitiva y real de los sectores de la ciudadanía que acaben perdiendo por la digitalización, frente a los sectores que ganen con la difusión de la inteligencia artificial y robótica, dependerá si los debates sobre el establecimiento de una renta básica universal, sobre la limitación pública de la inversión y difusión de las tecnologías 4.0 ('robotasas' por cada robot instalado), sobre el incremento de una fiscalidad más progresiva para proteger a quien pierde, se abrirán paso en la agenda social y política: "incluso aunque surjan nuevos empleos mientras los viejos se pierden por la automatización, será de poco consuelo para las personas que pierdan esos puestos de trabajo (...). Nuevos empleos que serán para otros trabajadores con cualificaciones tremendamente diferentes a las de los trabajadores despedidos" (Frey, 2019, p. 27).

Pragmáticamente, la automatización, la digitalización y la robotización redistribuyen las demandas de cualificación, las tareas de las ocupaciones, más que la eliminación del trabajo humano (Nedelkoska y Quintini, 2018): aquellos sin cualificación digital serán sustituidos y quienes sí la desarrollen, se integrarán cooperando con las tecnologías i40 (Hinojosa y Potau, 2017).

\section{(C) Automatización de integración: apostando organizativamente por una digitalización y robotización inclusivas mediante la recualificación del trabajo humano (otra vez):}

Un tercer enfoque, derivado de esa interpretación pragmática que rechaza la desaparición generalizada del trabajo humano por su sustitución digital, considera (y apuesta por) que la inteligencia artificial, la robótica y la 'industria 4.0' se implante y difunda mediante una automatización de integración entre las capacidades y fiabilidad de esta tecnología y las capacidades y fiabilidad humana de unos trabajadores y trabajadoras que pilotan, supervisan e intervienen en el funcionamiento de los dispositivos digitales y de los procesos digitalizados, así como en el diseño (y mejora continua humana) de sus algoritmos (explainable artificial intelligence$X A I^{\prime}$; Schüssler, 2020). A través de una cultura organizativa basada en un estilo tecnológico no únicamente de sustitución del factor humano, sino, por el contrario, en el reforzamiento e incremento en la intervención del factor humano en el control sobre la tecnología (augmentation automation, enabling technologies): la innovación tecnológica siempre será más eficaz y fiable, siguiendo una perspectiva ergonómica, si actúa coordinadamente con un factor humano cualificado, activo, con experiencia en los procesos productivos, con autonomía, con creatividad para mejorar continuamente los algoritmos digitales, traduciendo matemáticamente su experiencia en el 'lenguaje máquina' de programación, con su formación continua, con su participación: “Nuestro análisis nuestra que los humanos serán necesarios en la fuerza de trabajo: la ganancia de productividad total que estimamos vendrá si las personas trabajan junto a las máquinas" (McKinsey Global Institute, 2017, p. 3).

Esta integración amplía las capacidades del factor humano para que obtengan más resultados, para que desarrollen nuevas funciones, con más calidad y menos tiempo,

\footnotetext{
7 Para entender las potencialidades, sí, pero también las limitaciones y exageraciones de la inteligencia artificial es muy recomendable, como recurso gratuito accesible de formación para la ciudadanía, el curso abierto masivo online (MOoc) denominado Elements of AI, de la Universidad de Helsinki y Reaktor Education (https://www.elementsofai.com/).
} 
ampliando sus habilidades (Dellot, 2018; Ekbia y Nardi, 2017. Esta automatización de integración facilita la rentabilización más rápida, más eficaz y con más calidad de las fuertes inversiones de capital, de formación y de mantenimiento que exige (y ralentiza también) la introducción de la inteligencia artificial y la robótica, al tiempo que permite la mejora de las condiciones de trabajo del factor humano: "el conocimiento técnico mantiene su relevancia porque 'empodera' a los trabajadores para que ofrezcan una retroalimentación crítica sobre la implantación y el desarrollo continuado de los sistemas de IA, porque no todas y cada una de las posibles soluciones técnicas puede implantarse práctica y fiablemente" (Kolbe, 2020, p. 14). Eso sí, del factor humano cualificado, con conocimientos y competencias avanzados, lo que mantiene el tradicional debate sobre la polarización del futuro del trabajo humano y el empleo, entre los perdedores y los ganadores de la digitalización: mucho más cuando a los grupos profesionales semi y descualificados más afectados por la actual ola de automatización digital se les favorece hasta tres veces menos para participar en procesos formativos en sus organizaciones, frente a los perfiles ya ganadores de esos procesos (Nedelkoska y Quintini, 2018, p. 9).

La defensa de una automatización de integración implica devolver el protagonismo y la centralidad de los nuevos procesos de trabajo digitalizados al propio ser humano, a un factor humano que es considerado organizativamente como relevante, como un yacimiento de conocimientos tácitos sobre los procesos y las técnicas, de inteligencia y 'empirismo' aplicado, que permiten, si a ese factor humano se le proporcionan los suficientes recursos organizativos, la propia eficacia y efectividad de la inteligencia artificial: volviendo a hacer visible la importancia y el rol del factor humano para que la digitalización sea 'exitosa' y, por tanto, siga avanzando en su difusión. Apostando por una automatización, digitalización o robotización 'inclusiva' (Stewart et al., 2019; Degryse, 2016). Enfoque que permitiría equilibrar las prácticas mediáticas y empresariales en las que, cuando se presentan los nuevos prototipos de las tecnologías digitales de la i40, nuevos robots, nuevas aplicaciones, nuevos vehículos, nuevas instalaciones robotizadas, todo el foco se centra en la tecnología y sus 'habilidades', en muchas ocasiones 'ocultando' las redes de trabajadores y empleadas, de la fuerza de trabajo, que les dan 'soporte' para ese funcionamiento: como un mundo digital sin seres humanos, que es resultado del trabajo humano, velado tras las bambalinas organizativas (como el jugador de ajedrez humano escondido en las 'tripas' del autómata construido en 1770 por Wolfgang von Kempelen...).

\section{(D) HETEROMATIZACIÓN: HACIENDO VISIBLES LA IMPRESCINDIBLE COMPUTACIÓN HUMANA DEL TRABAJO HUMANO EN LA DIGITALIZACIÓN Y ROBOTIZACIÓN, TAMBIÉN SUS CONDICIONES DE TRABAJO:}

Visibilidad del trabajo y de los seres humanos que es el objetivo central de un último enfoque sobre la automatización digital y robotizada: el enfoque de la heteromatización (heteromation). Una propuesta de análisis de los procesos de digitalización que no proviene ni de las ciencias sociales, ni de la econometría, ni de las reflexiones jurídicas dominantes, sino de investigaciones de las ciencias de la computación, con una mirada etnográfica (sociológica y antropológica) sobre el funcionamiento real y organizativo de las tecnologías de automatización: subrayando no las expectativas (en ocasiones más cercanas a la 'ciencia-ficción', ¿tecno-fantasiosas?) de los prototipos, sino las experiencias reales de la inteligencia artificial ya implantadas, en funcionamiento. La heteromatización implica señalar que en muchos procesos digitales es imprescindible la participación del trabajo humano, la computación humana, para que funcionen adecuadamente: desde operadores humanos ${ }^{8}$ que etiquetan y/o aceptan imágenes, documentos y materiales en internet para que se incluyan en las bases de datos gestionadas bajo algoritmos (aburrida, rutinaria y estresante... al enfrentarse

8 'Digital janitors' y ‘netslaves'. 
El debate sobre la digitalización y la robotización del trabajo (humano) del futuro: automatización de sustitución, pragmatismo tecnológico, automatización de integración y heteromatización

habitualmente a imágenes violentas y brutales, que tienen que filtrar); 'etiquetaje' que no se puede automatizar por las dificultades (todavía) del reconocimiento de imágenes por la inteligencia artificial; pasando por el trabajo desarrollado en (micro)tareas para completar online servicios desmenuzados por parte de trabajadores y trabajadoras humanas en plataformas como Amazon Turk (Álvarez y Pérez Zapata, 2020); hasta el trabajo de los propios consumidores que gestionan aplicaciones digitales para realizar sus compras, realizando tareas 'gratuitamente' mediante el uso de las interfaces de las páginas para concluir la compra, o gestionando autónomamente sus productos en el uso de las cajas digitales sin 'empleo humano'... cuyo trabajo es realizado por los propios consumidores, manipulando los productos, tecleando y pulsando las pantallas táctiles, recogiendo los recibos (Dellot, 2018). Incluso en instalaciones industriales robotizadas, apoyadas por decenas de miles de trabajadores y trabajadoras, que nunca aparecen en las fotografías y vídeos de supuestas fábricas automáticas: por ejemplo, Tesla en su mítico centro de trabajo en Fremont (California $\left.{ }^{9}\right)$, supuestamente una fábrica automática y poshumana (Debord, 2017), mantiene, fuera del foco de la actividad de un centenar y medio de robots avanzados, casi una decena de miles de tradicionales trabajadores/as del automóvil que siguen trabajando en cadenas de montaje (con duras condiciones de trabajo, al tiempo que intentan sortear las políticas de la compañía para que no se sindiquen...; Wong, 2017).

Este soporte y trabajo humanos, aunque ocultados en las imágenes e imaginarios mediáticos sin seres humanos, son imprescindibles para que los sistemas digitales puedan funcionar como se espera, una instrumentalidad inversa (inverse instrumentality) que debe ser rastreada y hecha visible, puesto que la inteligencia artificial necesita apoyo humano para ser eficaz, para rellenar las lagunas que no puede cubrir en la complejidad de multitud de procesos productivos y de trabajo, todavía actualmente: una automatización realmente alimentada por el ser humano (human-fueled automation; Degryse, 2016). Es cuestionable que las tareas rutinarias no impliquen, además y como prerrequisito para su eficacia, simultáneamente tareas no-rutinarias, conocimientos y habilidades tácitas e implícitas que son en realidad difícilmente automatizables y que, por tanto, teniéndolas en cuenta en la investigación sociológica, explican un ritmo mucho más lento en la supuesta sustitución del factor humano por unas tecnologías digitales y robóticas, que siguen dependiendo de aquél (Pfeiffer, 2018a, 2018b).

El sistema tecnológico, realmente sus diseñadores/as humanos, sigue 'derivando' tareas al factor humano para su explotación, simulando que este último es innecesario: hacia "la capacidad computacional humana más barata que los sistemas automatizados" (Ekbia y Nardi, 2014). La heteromatización y su instrumentalidad inversa favorece el discurso del triunfo de la inteligencia artificial frente al trabajo humano enfatizando discursivamente que los sistemas digitales supuestamente proporcionan todo tipo de servicios a los seres humanos, cuando realmente están usando instrumentalmente el trabajo de éstos en múltiples tareas de apoyo y gestión: como sistemas digitales que requieren para ser funcionales al ser humano su capacidad de computación y acción humanas (Ekbia, 2016). En procesos que "combinan las capacidades de humanos y máquinas insertos en una narrativa de celebración de la máquina (...) Las organizaciones tienden a esconder su trabajo humano, empujándolo a los bastidores, narrando que las computadoras son las únicas que hacen el trabajo real. Esta estratagema tiende a hacer a los trabajadores y sus necesidades menos visibles, y a la organización menos responsable [respecto a esas necesidades]" (Nardi y Ekbia, 2018). Este enfoque de heteromatización permite también hacer emerger los costes humanos de esa computación humana, de la digitalización y de la inteligencia artificial. Como en la precarización de los riders que hacen funcionar el reparto logístico de bienes y servicios a través de las aplicaciones digitales de gestión de su trabajo humano, mientras son evaluados continuamente por la plataforma digital para la que trabajan: "Ocultar a

\footnotetext{
9 Que comenzó en 1962 como una fábrica de General Motors y posteriormente atrajo la atención de múltiples investigaciones de las ciencias del trabajo académicas entre 1995 y 2020, como ejemplo del diseño e implantación del toyotismo con su conversión para el nuevo modelo productivo conjunto entre GM y Toyota: NUMMI (New United Motor Manufacturing Inc).
} 
los trabajadores que están tras los formularios web, las API (Application Programming Interface) y las ATM (Active Media Technology) ayuda a los empleadores a verse a sí mismos como 'constructores' de tecnologías innovadoras, más que como empleadores despreocupados de sus condiciones de trabajo" (Irani y Six Silberman, 2013, p. 613).

\section{(E) BREVES CONCLUSIONES:}

La presentación de estos cuatro enfoques alternativos, pero complementarios, sobre la automatización con base en procesos de digitalización y robotización, mediante la implantación ampliada de la inteligencia artificial en un mayor número de procesos productivos y de prestación de servicios, permiten definir y diseñar estrategias de investigación para analizar y evaluar sus efectos, por ejemplo, en las condiciones de empleo y en las condiciones de trabajo.

Sin embargo, por su origen exclusivamente econométrico, las perspectivas de la automatización de sustitución y el enfoque pragmático se centran exclusivamente en predecir cuantitativamente los posibles efectos en la cantidad de empleo futuro, sin abordar realmente cuáles son las características de las tecnologías de la i40, sus acciones y funcionamiento, los contenidos técnicos y organizativos de las tareas concretas que son automatizadas, digitalizadas y robotizadas, los márgenes de actuación del factor humano, lo que, metodológicamente, dificulta o debilita anticipar si una tecnología va a sustituir una ocupación (total o parcialmente), modificarla con más o menos intensidad, crear otras nuevas ocupaciones, tareas o perfiles profesionales, a pesar de su 'impacto' mediático. Eso sí, ambas permiten acceder, explicar y comprender los discursos 'tecno-optimistas/ pesimistas' que sustentan mediáticamente, haciéndolos socialmente (y sociológicamente) relevantes, la difusión de los imaginarios del fin del trabajo y del empleo humanos.

Por el contrario, tanto el enfoque de automatización de integración como de heteromatización, originados en las ciencias sociales y del comportamiento (psicosociologías del trabajo, ergonomía, etnografías antropológicas...) y en las ciencias de la computación aplicadas, se enfocan prioritariamente a conocer e intervenir en las condiciones organizativas reales con las que se implanta la inteligencia artificial y la robótica, preocupadas más en la calidad de las condiciones de actividad de la interacción del factor humano con esas nuevas tecnologías digitales: con el objetivo de hacer visibles los efectos (diseñados y construidos socialmente con base en un estilo tecnológico de automatización; Lahera Sánchez, 2006) sobre la eficacia, la eficiencia de la organización y, sobre todo, en relación con la salud laboral, a la adquisición o pérdida de cualificaciones, a la mejora o deterioro de las condiciones de trabajo, a la emergencia de nuevos riesgos laborales a eliminar o controlar.

Sin olvidar la redistribución de las ganancias de productividad que se pueden obtener con la automatización digital en empresas y organizaciones ${ }^{10}$ : “Una cuestión crucial es si la ganancia de productividad de las nuevas tecnologías conlleva a beneficios más altos para las empresas, a precios más bajos para los consumidores o a salarios más altos para los trabajadores. Si hubiera realmente unas significativas ganancias de productividad en el futuro, estas serán el asunto fundamental de la política de distribución" (Eurofound, 2019, p. 3). Cómo se negocie esa redistribución de la productividad, cómo se estratifique socialmente entre los perdedores y los ganadores de los actuales procesos de automatización, cómo se luchen o se acepten sus efectos laborales, conforman realmente el problema sociológicamente (y políticamente) más relevante de unas tecnologías digitales que, aunque sea más lentamente de lo publicitado, seguirán con nosotros en el futuro, definiendo las condiciones de vida y empleo de la ciudadanía.

\footnotetext{
10 Lo que obliga a las organizaciones sindicales también a conocer las características de las tecnologías de la Cuarta Revolución Industrial, sus contenidos, las transformaciones que pueden provocar en las competencias, tareas y cualificación de las fuerzas de trabajo a las que representan en la gestión de las relaciones laborales: a través de propuestas de 'Sindicalismo 4.0', por ejemplo (Lahera Sánchez, Negro y Tovar, 2019). Sin olvidar que la presencia sindical en sectores y perfiles profesionales más vanguardistas en la digitalización es muy limitada...
} 
El debate sobre la digitalización y la robotización del trabajo (humano) del futuro: automatización de sustitución, pragmatismo tecnológico, automatización de integración y heteromatización

\section{REFERENCIAS}

Acemoglu, D.; Restrepo, P. (2017). Robots and Jobs. Evidence from U.S. Labor Markets. National Bureau of Economic Research, Working Paper 23285.

Allen, R. (2007). Engel's Pause: A Pessimist's Guide to the British Industrial Revolution, Economics Series Working Papers 315, University of Oxford.

Álvarez, G. y Pérez Zapata, O. (2020). Hacia la plataformización: el caso de una plataforma digital cualificada. En A. Riesco, A. (Ed.), Fronteras del trabajo asalariado. Madrid: La Catarata.

Arntz, M., Gregory, T. y Zierahn, U. (2016). The Risk of Automation for Jobs in OECDcountries: A Comparative Analysis. OCDE Social, Employment \& Migrations Working Papers, 189.

Benanav, A. (2019). Automation and the future of work. New Left Review, 119-120.

Brynjolfsson, E. y McAfee, A. (2011). Race Against the Machine: How the Digital Revolution is Accelerating Innovation, Driving Productivity, and Irreversibly Transforming Employment and the Economy. Mass: Digital Frontier Press.

Brynjolfsson, E. y McAfee, A. (2015). The Second Machine Age: Work, Progress and Prosperity in a Time of Brilliant Technologies. Nueva York: W.W. Norton \& Company.

Canales, K. (2020). Some of San Francisco's robot-run restaurants are failing. It could simply be that we still want to be served by humans, not machines. Business Insider, 12 de enero de 2020. https://www.businessinsider.com/san-francisco-robot-restaurants-failingeatsa-cafex-2020-1

Fundación COTEC (2020). III Encuesta de Percepción Social de la Innovación en España. Madrid: Fundación COTEC para la Innovación y Sigma Dos.

Debord, M. (2017). Tesla's future is completely inhuman - and we shouldn't be surprised". Business Insider, 20 de mayo de 2017. https://static2.businessinsider.com/teslacompletely-inhuman-automated-factory-2017-5

Degryse, Ch. (2016). Digitalisation of the economy and its impact on labor markets. European Trade Unions Institute-ETUI Working Paper 2016.02.

Dellot, B. (2018). Why automation is more than just a job killer? RSA blog, Royal Society for the Encouragement of Arts, Manufactures and Commerce. https://www.thersa.org/ blog/2018/07/the-four-types-of-automation-substitution-augmentation-generationand-transference

Doménech, R., García, J.R., Montáñez, M. y Neut, A. (2018). ¿Cuán vulnerable es el empleo en España a la revolución digital? BBVA Research: Observatorio Económico.

Ekbia, H. y Nardi, B. (2017). Heteromation and Other Stories of Computing and Capitalism. Cambridge: MIT Press.

Ekbia, H. y Nardi, B. (2014). Heteromation and its (dis)contents: the invisible division of labor between humans and machines. First Monday, 19 (6).

Ekbia, H.R. (2016): Digital inclusion and social exclusion: the political economy of value in a networked world. The Information Society, 32 (3), 165-175.

Eurofound (2019). The Future of Manufacturing in Europe. Luxemburgo: Publications Office of the European Union.

Ford, M. (2015). The Rise of Robots. Londres: Oneworld Publications. 
Frey, C.B. (2019). The Technological Trap. Capital, Labor and Power in the Age of Automation. Princeton: Princeton University Press.

Frey, C.B. y Osborne, M.A. (2013). The Future of Employment: How Susceptible are Jobs to Computerization? Working Paper, Oxford Martin School, University of Oxford, 77 páginas.

Hidalgo, M.A. (2018). El empleo del futuro. Un análisis del impacto de las nuevas tecnologías en el mercado laboral. Barcelona: Deusto.

Hinojosa, C. y Potau, X. (2017). Advanced Industrial Robotics: Taking human-robot collaboration to the next level. Eurofound, WPFOMEEF 18003.

International Federation of Robotics (2018). Robots and the workplace of the future. IFR Positioning Paper.

Irani, L. y Six Silberman, M. (2013). Turkopticon: Interrupting worker invisibility in Amazon Mechanical Turk. Annual CHI Conference on Human Factors in Computing System 2013.

Klenert, D., Fernández-Macías, C. y Antón, J.I. (2020). Do robots really destroy jobs? Evidence from Europe. Joint Research Center-European Commission, JCR 118393.

Kolbe, D. (2020). Into a new era of work. En Social Europe: Artificial Intelligence. Berlín: Social Europe Publishing, 10-15.

Lahera Sánchez, Arturo (2006). Diseño tecnológico y proceso de trabajo: mutaciones organizativas en empresas de ingeniería mecánica. Madrid: Miño y Dávila Editores.

Lahera Sánchez, Arturo (2019). Digitalización, robotización, trabajo y vida: Cartografias, debates y prácticas. Cuadernos de Relaciones Laborales, 37 (2), 249-273.

Lahera Sánchez, A., Negro, A. y Tovar, F.J. (2019). Sindicalismo 4.0 y negociación tecnológica: Por un diseño integrador de los procesos de digitalización y robotización. Comunicación para el Congreso Interuniversitario sobre el Futuro del Trabajo, Organización Internacional del Trabajo.

Lobera, J. y Torres-Albero, C. (Eds.) (2019): Percepción social de la Ciencia y la tecnología 2018. Madrid: Fundación Española para la Ciencia y la Tecnología-FECYT.

Lobera, J., Fernández Rodríguez, C.J. y Torres-Albero, C. (2020): Privacy, Values \& Machines: Predicting Opposition to Artificial Intelligence. Communication Studies, 71(3), 448-465.

McKinsey Global Institute (2017). A Future that Works: Automation, Employment, and Productivity. Londres: McKinsey \& Company.

Mishel, L. y Bivens, J. (2017). The Zombie Robot Argument Lurches On: There is no evidence that automation leads to joblessness or inequality. Economic Policy Institute, report 126750.

Nardi, B., Ekbia, H. (2018). The future of human labor: A look at manufacturing and war. ACMAssociation for Computing Machinery SIGCAS Computer \& Society, 47 (4), 46-53.

Nedelkoska, L. y Quintini, G. (2018). Automation, skills use and training. OECD Social Employment \& Migration Working Papers, № 202.

Ortega, A. (2016). La imparable marcha de los robots. Madrid: Alianza Editorial.

Pfeiffer, S. (2018a). Industry 4.0: Robotics and Contradictions. En Bilić P., Primorac J.y Valtýsson B. (Eds), Technologies of Labour and the Politics of Contradiction. Dynamics of Virtual Work (pp. 19-36). Londres: Palgrave Macmillan.

Pfeiffer, S. (2018b). The 'Future of Employment` on the Shop Floor: Why Production Jobs are Less Susceptible to Computerization than Assumed. International Journal for Research in Vocational Education and Training, 5(3), 208-225. 
El debate sobre la digitalización y la robotización del trabajo (humano) del futuro: automatización de sustitución, pragmatismo tecnológico, automatización de integración y heteromatización

PriceWaterhouseCoopers (2018). Will robots really steal our jobs? PWC documents.

Scholten, Ch. (2017). Industrial Internet of Things: Digitisation, value networks and changes in work. Eurofound WPFOMEEF 18006.

Serrano, A. y Fernández Rodríguez, C. J. (2018). De la metáfora del mercado a la sinécdoque del emprendedor: la reconfiguración política del modelo referencial de trabajador. Cuadernos de Relaciones Laborales, 36(2), 207-224.

Schüssler, M. (2020): Explaining Artificial Intelligence in Human-Centred Terms. En Social Europe: Artificial Intelligence. A Social Europe Dossier. Berlín: Social Europe Publishing.

Stewart, K., Deshpande, A., Hoorens, S. y Gunashekar, S. (2019). Advanced robotics. Implications of game-changing technologies in the service sectors. Eurofound Working Paper WPEF19001.

Susskind, D. (2020). A World without Work. Technology, Automation and How we should respond. Nueva York: Henry Holt \& Company.

Van Barneveld, J. y Janson, T. (2017). Additive Manufacturing: A layered revolution. Eurofound Working Paper WPFOMEEF 18002.

Wong, J.C. (2017). Tesla factory workers reveal pain, injury and stress: 'Everything feels like the future but us'. The Guardian, 17 de mayo de 2017. https://www.theguardian.com/ technology/2017/may/18/tesla-workers-factory-conditions-elon-musk

World Economic Forum (2016). The Future of Jobs. Employment, Skills and Workforce Strategy for the Fourth Industrial Revolution. Ginebra: World Economic Forum.

World Economic Forum (2018). The Future of Jobs Report 2018. Ginebra: World Economic Forum.

\section{NOTA BIOGRÁFICA}

Arturo Lahera Sánchez es Profesor de Ergonomía y Sociología del Trabajo en la Universidad Complutense de Madrid (alaheras@ucm.es). Investigador principal del proyecto FINDeR-Futuros del Trabajo: Digitalización, Robotización e Industria 4.0 (Fondo Específico de Investigación UCM: FEI-EU-17-25). Responsable del área de Sociología del Trabajo del proyecto FuWorkTech-El impacto de la digitalización en las relaciones de trabajo: retos y oportunidades (Ministerio de Ciencia e Innovación: PID2019104287RB-100; Proyectos de I+D+i RTI Tipo B); y miembro del equipo de investigación ROVIN-Robotización y transformaciones del trabajo en el sector vitivinícola (Universidad de Valladolid). 